\title{
Don't Let a Crisis Go to Waste
}

\author{
Winta Mehtsun, MD, MPH, and Kenneth Tanabe, MD \\ Division of Gastrointestinal and Oncologic Surgery, Massachusetts General Hospital, Boston, MA
}

It has been longer than a year since the first case of SARS-CoV-2 was reported in December 2019. At time of this writing, reports show more than 122,086,638 confirmed cases of the disease caused by SARS-CoV-2, coronavirus disease 2019 (COVID-19), and 2,695,945 deaths globally. ${ }^{1}$ The death toll of the COVID-19 pandemic in the United States alone surpasses the number of Americans killed in World War II, Korea, and Vietnam combined. Nothing else in our lifetime has had such widereaching and devastating consequences.

Health systems have been hit hard. Juggling the capacity demands of COVID-19 patients and triaging care for nonCOVID-19 patients while trying to maintain safety for patients and providers have been extremely challenging, and for some health systems, frankly, unachievable. At the height of the first wave of the pandemic, hospitals canceled elective operations as a strategy to increase critical care capacity, preserve personal protective equipment (PPE), and deploy surgical staff to support care for COVID-19 patients. The pandemic has required hospitals and health networks to redesign their treatment algorithms for nonelective complex conditions such as cancer while in parallel addressing the immediate threats of COVID-19. Several surgical and medical oncology societies across the world developed triage recommendations for cancer patients, with many developing a priority-based approach to cancer care that weighed curative potential against risk of treatment delay. ${ }^{2-4}$ The toll of the COVID-19 pandemic on cancer care still is evolving, but early evidence already suggests that patients across the globe have experienced

(C) Society of Surgical Oncology 2021

First Received: 3 May 2021

Accepted: 4 May 2021;

Published Online: 16 June 2021

K. Tanabe, MD

e-mail: ktanabe@partners.org interruption in cancer-specific care. ${ }^{5}$ With 1 year of the pandemic behind us, optimism is in sight. Several vaccines have been developed and are getting into the arms of our patients. Our understanding of the transmission and treatment for COVID-19 has improved. Yet it is clear that we are still a long way from pre-pandemic norms.

The goal of providing timely and safe oncologic care during the pandemic remains a moving target. Initially, we were forced to develop real-time solutions in resourceconstrained environments, with little understanding of the risks our decisions posed for patients and health care providers. Two articles in this issue seek to address some of this uncertainty by examining the safety of treatment with gastroesophageal surgery during the early phase of the COVID-19 pandemic. ${ }^{6,7}$ Both studies took place in European referral centers with varying rates of community prevalence and across a spectrum of national lockdown and social-distancing measures.

Borgstein et al. ${ }^{6}$ examine the safety of esophageal surgery for patients during the COVID-19 pandemic across four European tertiary referral centers in the Netherlands, Germany, Belgium, and Sweden. Their primary end point of interest, the rate of respiratory failure requiring mechanical ventilation, was compared between a COVID19 pandemic cohort (1 March 2020 to 31 May 2020) and a historical cohort (1 October, 2019 to 29 February 2020).

The authors identified 139 patients in the COVID-19 cohort and 168 patients in the historic control cohort and found no difference between them in the rate of respiratory failure requiring mechanical ventilation (13.7 vs 8.3; $p=0.127)$ and the number of pulmonary complications $(32.4 \%$ vs $29.9 \%)(p=0.646)$. In the COVID-19 cohort, 134 of the patients $(71.9 \%)$ had a preoperative reverse transcription-polymerase chain reaction (RT-PCR), and only $25.9 \%$ of the patients had a postoperative RT-PCR. The timing of this initial PCR with regard to admission for surgery is unclear. No association was found between 
undergoing esophageal surgery in the COVID-19 pandemic and postoperative respiratory failure requiring mechanical ventilation.

Alasmar et al. ${ }^{7}$ examined the safety of gastroesophageal surgery during the COVID-19 pandemic between 1 May and 1 July 2020 across nine European specialty centers in the United Kingdom, Italy, Spain, Belgium, and the Netherlands. The primary end point for their study was the 30-day COVID-19 mortality rate for the patients undergoing gastroesophageal surgery. In an effort to identify patient-to-staff transmission, health care providers giving patient care in the operating room were invited to complete a health survey to determine the incidence of COVID-19 infection among the medical staff.

The authors also examined the types of precautions taken across the hospitals included in the study. Only one hospital was COVID-19-free, but most had some degree of COVID-19-free pathways postoperatively. However, it was unclear the degree to which the providers were truly isolated from cross-contamination between these COVID-19free units and COVID-19 units. The preoperative RT-PCR policies for the patients also were variable, ranging from no testing at one facility to testing $24-96 \mathrm{~h}$ preoperatively. The extent and frequency of staff testing also were not well described beyond binary descriptions (yes, no, or some). Similarly, the type of PPE used by the staff in the operating room was not consistent across hospitals, with no specification about re-use and prevalence of use among all hospital personnel.

Among 159 patients examined during the study period, preoperative COVID testing was completed for 149 patients $(93.7 \%)$, and 2 patients $(1.3 \%)$ were found to be positive for COVID, requiring a delay in surgery until a negative test. Postoperatively, 39 patients $(24.7 \%)$ underwent postoperative RT-PCR tests for suspected COVID infection, none of which was positive. One death was reported involving a patient with a negative COVID test. Of the 403 eligible staff, 313 (78\%) completed the COVIDrelated health survey. Among the responders, 2 providers $(0.6 \%)$ from the same hospital tested positive for COVID during the study period.

Both studies concluded that gastroesophageal surgery can be safely performed during a high prevalence of community COVID-19. Borgstein et al. ${ }^{6}$ found no difference in the rates of postoperative respiratory failure requiring mechanical ventilation (or any of the other secondary end points) between the patients who underwent gastroesophageal surgery during the first wave of the COVID-19 pandemic and the historic control patients. Alasmar et al. ${ }^{7}$ found a low incidence of COVID-19 among patients and providers and no COVID-related mortality among patients.
Both studies also drew the conclusion that because a majority of patients underwent minimally invasive procedures without untoward complications, the strategy of performing minimally invasive surgery during the pandemic is safe. However, to state such a conclusion regarding the potential of increased SARS-CoV2 transmissibility with minimally invasive approaches, robust RTPCR-testing of patients and all providers involved in the operative care of the patient must be performed. This should include access to granular data regarding timing and frequency of RT-PCR-testing because we know the sensitivity of the test is approximately 70\%. Although confidence in the conclusions reached by both studies is limited by the small sample size, selection bias, a focus on short-term outcomes, and absence of details on the timing and frequency of COVID-19 RT-PCR-testing, these articles still bring the following meaningful and practical takeaways to light:

1. Delivering optimal cancer care during the COVID-19 pandemic requires visionary leadership, strong administrative competence, adept resource allocation, and optimal patient selection.

The implementation of protocols to maintain patient and provider safety during a pandemic of this magnitude requires leadership, expertise, and resources, which are not equally available across all hospitals and health systems. Clearly, the centers highlighted in both studies had the necessary leadership, administrative competence, and resources to deliver cancer care amid the pandemic with reasonable short-term outcomes. Appropriate patient selection was a fundamental part of the success their programs achieved. Policies and guidelines evolved in real time and required reassessment to ensure that patient and provider safety objectives were continually met with the learning of new data. More insight into their allocation and triage strategies would be helpful to the gathering of best practices in the event of a similar crisis.

2. The rates of nosocomial SARS-CoV2 infection are low, but we must continue to remain vigilant and reassure patients that seeking out facility-based health care can be safe.

At the height of the pandemic, many patients avoided treatment for essential conditions for fear of contracting SARS-COV2. Despite the ground-breaking progress in vaccine development and an improved understanding of SARS-CoV-2 infection and its treatment, many patients still are more comfortable going to the grocery store than they are seeking out cancer care in a hospital or outpatient setting. Although both studies found a low incidence of COVID positivity among patients undergoing 
gastroesophageal surgery, the timing and frequency of testing were not clearly delineated. Thus, it is hard to delineate whether the rate of asymptomatic SARSCoV-2 infection was underestimated in both studies. Larger studies published subsequently have found low rates of nosocomial infection in hospitals due to the evolution of rigorous infection control practices. ${ }^{8}$

Sporadic outbreaks in hospitals can occur. A subsequent study investigating a SARS-CoV-2 infection among patients and hospital staff at the Brigham and Women's Hospital identified a cluster originating from a patient with unrecognized COVID-19 infection. ${ }^{9}$ Certain factors associated with transmission included nebulization of the index case, positive pressure rooms, staff caring for patients with dyspnea or cough, exposure to a case patient longer than 15-min, lack of eye protection, and interaction with SARS-CoV-2positive staff in clinical work areas. The most recent changes in infection control procedures implemented in the Brigham study were enhanced eye protection for employees, universal testing at admission, daily screening of nurses for COVID-19 symptoms, and a hospital-wide shift to N95 masks for routine COVID19 care. With increased testing capacity and adequate PPE now increasingly available at tertiary referral centers, rates of nosocomial infection can remain extremely low with vigilant adherence to infection control protocols.

3. Cancer care in a post-COVID-19 pandemic world needs to evolve to eliminate low-value care.

Nothing exposes redundancy and inefficiency more than a crisis. Multi-disciplinary oncology task forces assembled across the world to adapt practice patterns at various time points in the COVID-19 pandemic that incorporated digital pre-screening clinics, shifted inperson visits to telemedicine visits, altered multimodality algorithms when appropriate, and re-triaged surgical delays. ${ }^{4}$ Consequently, we likely will reconsider how much of oncologic care needs to be within the walls of a health facility. That is, the use of telehealth for routine postoperative visits and systemic therapy surveillance likely will be the norm instead of the exception. As we imagine a post-pandemic world, it is clear that health systems need to reconsider cancer care delivery models and share successful modifications across various practice environments. ${ }^{10}$

DISCLOSURES There are no conflicts of interest.

\section{REFERENCES}

1. COVID-19 Dashboard. The Johns Hopkins Coronavirus Resource Center. Retrieved 18 March 2020 at https://coronavirus.jhu.edu/ map.html.

2. Hwang ES, Balch CM, Balch GC, et al. Surgical oncologists and the COVID-19 pandemic: guiding cancer patients effectively through turbulence and change. Ann Surg Oncol. 2020;27:2600-13. http s://doi.org/10.1245/s10434-020-08673-6.

3. Collaborative $\mathrm{CO}$. Global guidance for surgical care during the COVID-19 pandemic. Br J Surg. 2020;107:1097-103. https://doi. org/10.1002/bjs.11646.

4. Cinar P, Kubal T, Freifeld A, et al. Safety at the time of the COVID19 pandemic: how to keep our oncology patients and healthcare workers safe. J Natl Compr Canc Netw. 2020;18(5):1-6. https://d oi.org/10.6004/jncen.2020.7572.

5. Jazieh AR, Akbulut H, Curigliano G, et al. Impact of the COVID19 pandemic on cancer care: a global collaborative study. JCO Glob Oncol. 2020;6:1428-38. https://doi.org/10.1200/GO.20. 00351.

6. Borgstein ABJ, Brunner S, Hayami M, et al. Safety of esophageal cancer surgery during the first wave of the COVID-19 pandemic in Europe: a multicenter study. Ann Surg Oncol. 2021. https://doi. org/10.1245/s10434-021-09886-Z.

7. Alasmar M, Afsana Kausar A, Berend-Jan Borgstein A, et al. Is re-introducing major open and minimally invasive surgery during COVID-19 safe for patients and healthcare workers? An international, multi-center cohort study in the field of esophagogastric surgery. Ann Surg Oncol. 2021. https://doi.org/10.1245/ s10434-021-09885-0.

8. Rhee C, Baker M, Vaidya V, et al. Incidence of nosocomial COVID-19 in patients hospitalized at a large US academic medical center. JAMA Netw Open. 2020;3:2020498. https://doi. org/10.1001/jamanetworkopen.2020.20498.

9. Klompas M, Baker MA, Rhee C, et al. A SARS-CoV-2 cluster in an acute care hospital. Ann Intern Med. 2021. https://doi.org/10. 7326/M20-7567.

10. Gyawali B, Poudyal BS, Eisenhauer EA. Covid-19 pandemic-an opportunity to reduce and eliminate low-value practices in oncology? JAMA Oncol. 2020;6(11):1693-94. https://doi.org/10. 1001/jamaoncol.2020.2404.

Publisher's Note Springer Nature remains neutral with regard to jurisdictional claims in published maps and institutional affiliations. 